\title{
Factores sociológicos y calidad de vida relacionada con la salud en pacientes en hemodiálisis
}

José Luis Cobo Sánchez ${ }^{1}$ - Raquel Pelayo Alonso² - Emilio Ibarguren Rodríguez ${ }^{2}$ - Ainhoa Aja Crespo ${ }^{2}$ Araceli Saenz de Buruaga Perea ${ }^{2}-M^{a}$ Elena Incera Setién ${ }^{2}-M^{a}$ Yolanda Vicente Jiménez ${ }^{2}$ - Rosa Ana Sainz Alonso ${ }^{2}$ - Lucía Merino González ${ }^{2}$ - Ana Isabel Serrano Rubio ${ }^{2}$ - Ana Isabel Pérez Garmilla ${ }^{2}$ Rosa Alonso Nates ${ }^{2}$

${ }^{1}$ Máster en Ciencias de la Enfermería

${ }^{2}$ Diplomado en Enfermería

Servicio de Nefrología. Hospital Universitario Marqués de Valdecilla. Santander

\section{Resumen}

Objetivo: Evaluar la influencia de factores sociológicos sobre la calidad de vida relacionada con la salud percibida por los pacientes en hemodiálisis de nuestra unidad.

Material y método: Estudio prospectivo, descriptivo transversal sobre pacientes en hemodiálisis que hubiesen permanecido al menos 3 meses en tratamiento como crónicos. Como instrumento de medida se utilizó el Euroqol-5D. Como variables sociológicas dependientes se estudiaron: edad, sexo, estado civil, convivencia, nivel de estudios y nivel de ingresos mensuales.

Resultados: Se estudiaron 47 pacientes: $67 \%$ fueron hombres, con una edad media de $62 \pm 15$ años. El $63 \%$ padecía algún tipo de dolor/malestar moderado, y el $49 \%$ estaba ansioso/deprimido en distinto grado. Ni el sexo ni el nivel de estudios se correlacionó con ningún parámetro del Euroqol-5D. Los casados presentaron peores puntuaciones en cuanto a la EVA $(p=0,012)$, ansiedad/depresión $(p=0,002)$ y percepción del estado de salud en el último año $(p=0,016)$. Los que vivían solos presen-

\footnotetext{
Correspondencia:

José Luis Cobo Sánchez

Servicio de Nefrología
}

Hospital Universitario Marqués de Valdecilla

Avda. Valdecilla s/n 39008 Santander. Cantabria

E-mail: jlcobosanchez@gmail.com taron mejores puntuaciones en la EVA $(p=0,062)$ y menor dolor/malestar $(p=0,075)$. El nivel de ingresos se correlacionó inversamente con problemas en el aseo personal $(p=0,016)$. Los mayores de 65 años presentaban peor percepción de su estado de salud que los menores de 65 años $(p=0,006)$.

Conclusiones: Casi el $70 \%$ de los pacientes presenta dolor/malestar y casi la mitad se encuentra ansioso o deprimido. Las variables sociológicas que más influyen en la calidad de vida relacionada con la salud de los pacientes en hemodiálisis son la edad, el estado civil, la convivencia y el nivel de ingresos.

PALABRAS CLAVE:

- CALIDAD DE VIDA

- HEMODIÁLISIS

- INDICADORES SOCIALES

- CUESTIONARIOS

Sociological factors and health related quality of life to in hemodialysis patients.

\section{Abstract}

Objective: To evaluate the influence of sociological factors on the perceived health related quality of life among hemodialysis patients in our unit. 
Methodology: Prospective, descriptive and crosssectional study in hemodialysis patients who had been at least 3 months on chronic treatment. EuroQol-5D was used as a measuring instrument. As sociological dependent variables were studied: age, sex, marital status, cohabitation, educational level and monthly income.

Results: Forty-seven patients were studied: $67 \%$ men, mean age $62 \pm 15$ years. Sixty-three percent suffered some type of moderated pain / discomfort, and $49 \%$ were anxious / depressed to varying degrees. Neither sex nor educational level correlated with any parameter of the EuroQol-5D. Married people had worse scores in terms of VAS $(p=0.012)$, anxiety / depression $(p=0.002)$ and perceived health status in the past year $(p=0.016)$. Those who lived alone had better scores on the VAS $(p=0.062)$ and less pain / discomfort $(p=0.075)$. Income level was inversely correlated with personal hygiene problems $(p=0.016)$. Patients over 65 years had poorer perception of health status that patients under 65 years $(p=0.006)$.

Conclusions: Nearly $70 \%$ of patients have pain / discomfort, and nearly half are anxious or depressed. Sociological variables that influence the quality of life related to health in hemodialysis patients are age, cohabitation, marital status and income level.

\section{KEY WORDS:}

- QUALITY OF LIFE

- HEMODIALYSIS

- SOCIAL INDICATORS

QUESTIONNAIRES

\section{Introducción}

El concepto de calidad de vida relacionada con la salud (CVRS) aparece en las sociedades occidentales en un momento en que la esperanza de vida ha aumentado sensiblemente y en el que predomina la convicción de que el papel de la medicina no debe ser únicamente el de proporcionar muchos años de vida a las personas, sino, sobre todo, el de aportar una mejora en la calidad de los años vividos ${ }^{1}$.
Para ser más precisa la CVRS, designa los juicios de valor que un paciente realiza acerca de las consecuencias de su enfermedad o tratamiento. Es un concepto unipersonal, multideterminado (actitud frente a la vida, creencias, actividad laboral, etc.) y que no siempre coincide con las estimaciones realizadas por el médico ${ }^{1}$. La OMS define calidad de vida como la "percepción del individuo de su posición en la vida en el contexto de la cultura y sistema de valores en los que vive y en relación con sus objetivos, expectativas, estándares y preocupaciones ${ }^{\prime \prime 2}$. Las personas que padecen una enfermedad crónica como la enfermedad renal crónica suelen estar más interesadas en conseguir la mejor calidad de vida posible que en buscar la curación, ya que saben que quizás tengan que vivir mucho tiempo con la enfermedad².

Los pacientes que deben someterse a tratamientos sustitutivos altamente invasivos involucran cambios de vida en los ámbitos físico, psicológico y social para el paciente y su familia. Las diferentes modalidades de tratamiento renal sustitutivo (hemodiálisis y diálisis peritoneal), tienen diferentes repercusiones en las personas ${ }^{3}$. Para las personas en hemodiálisis (HD), la diálisis se convierte en el eje de su vida, implicando grandes restricciones. Las personas tratadas con diálisis peritoneal tienen más flexibilidad en horarios y menos restricciones para los desplazamientos, pudiendo alcanzar una mayor compatibilidad con otras responsabilidades. La diálisis conlleva cambios adversos, ante los cuales las personas ponen en marcha pensamientos y acciones para afrontarlos, unos consiguen encontrar una vida más o menos satisfactoria mientras que otros se sumen en el pesimismo y el abandono. Estas diferencias se pueden explicar por las Ilamadas estrategias que Lazarus y Folkman han definido como los esfuerzos cognitivos y conductuales constantemente cambiantes que un individuo realiza para "manejar" las demandas externas e internas y que son evaluadas como algo que agrava o excede los recursos de la persona ${ }^{4}$.

Algunos estresores en la vida de los pacientes en HD pueden causar cambios en diferentes contextos del estado de los pacientes, como la vida familiar, marital, social u ocupacional. Algunos de estos estresores son: efectos de la enfermedad, dinámicas familiares, restricciones de la dieta, horarios, limitaciones funcionales, limitaciones económicas, cambios en el empleo, relaciones con el personal de $\mathrm{HD}$, cambios en el rol, cambios en la función sexual, efectos de la medicación y conocimientos para afrontar la muerte ${ }^{5}$. 
El objetivo de este estudio fue evaluar la influencia de factores sociológicos sobre la CVRS percibida por los pacientes en HD de nuestra unidad.

\section{Pacientes y método}

Estudio prospectivo, descriptivo transversal sobre pacientes en HD crónica. Como criterio de inclusión se fijó que los pacientes hubiesen permanecido al menos 3 meses en tratamiento con HD como crónicos. Se excluyeron a aquellos pacientes que su estado cognitivo no les permitía contestar el instrumento de medida de CVRS. El instrumento utilizado para evaluar la CVSR fue el Euroqol-5D (EQ-5D): se trata de un cuestionario autoadministrado, validado para población española por Badia y cols ${ }^{6}$ y utilizado con anterioridad para evaluar la CVRS en pacientes en $H^{7-9}$. EI EQ-5D es una medida genérica multidimensional de la CVRS que puede expresarse como un perfil del estado de salud o como un valor índice. Consta esencialmente de dos partes:

1) La primera de ellas, es una descripción del estado de salud en cinco dimensiones: movilidad, cuidado personal, actividades cotidianas, dolor/malestar y ansiedad/depresión. Cada una de estas dimensiones tiene tres ítems que definen tres niveles de gravedad: ningún problema, algunos problemas y muchos problemas. El individuo señala el nivel de cada dimensión que le es aplicable en el "día de hoy".

2) En la segunda parte, el individuo puntúa su estado de salud en una escala visual analógica milimetrada o termómetro (EVA) de $20 \mathrm{~mm}$ cuyos extremos son 0 : peor estado de salud imaginable y 100: mejor estado de salud imaginable. El individuo señala la puntuación que otorga a su estado de salud en el "día de hoy". El valor asignado en la EVA puede ser usado como indicador cuantitativo del estado general de salud de un individuo.

Agregamos al instrumento una tercera parte, en la que se pedía al entrevistado que evalúe su estado de salud con respecto a los últimos 12 meses como igual, mejor o peor.

Como variables sociológicas dependientes se estudiaron la edad, sexo, estado civil (soltero, casado, viudo, divorciado, en unión/pareja estable), convivencia (familia, solos, en institución sociosanitaria), nivel de estudios (analfabeto, sin estudios, primer grado - estudios primarios o cinco cursos aprobados de EGB o equivalentes-, segundo grado - titulo de Bachiller elemental, Graduado escolar, EGB completo, FP de $1^{\circ}$ y $2^{\circ}$ grado, Bachiller superior-, tercer grado-Titulaciones de Escuelas Técnicas Superiores, Diplomados de Escuelas Universitarias y Diplomados de Facultades o Colegios Universitarios, Licenciatura-) y nivel de ingresos mensuales $(<500 €$, entre 500 y $1000 €$, entre 1000 y $1500 €$, entre 1500 y $2000 €$ y $>2000 €)$.

EI EQ-5D y el cuestionario respecto a las variables sociológicas fue autocumplimentado durante la sesión de HD de forma anónima. Se solicitó el consentimiento informado a todos los pacientes que participaron en el estudio.

El análisis estadístico se realizó con el paquete estadístico SPSS v.o. 15.0 para Windows. Para la inferencia estadística se aplicaron los tests de Chi cuadrado y Kruskal-Wallis. Los resultados se consideraron significativos si el nivel crítico observado era inferior al $5 \%(p<0,05)$.

\section{Resultados}

De los 50 pacientes de nuestra unidad, se excluyeron 7 porque su estado cognitivo no les permitía contestar al cuestionario empleado. De los 47 pacientes estudiados, el $67 \%$ fueron hombres y el $33 \%$ mujeres, con una edad media de 62 \pm 15 años (rango 30-88 años). El tiempo medio en HD fue de $49 \pm 50$ meses (rango 12-229 meses). Las nefropatías más comunes fueron la vascular, glomerular y sistémica (23\% respectivamente), seguidas de la diabética (13\%) y la intersticial y desconocida ( $9 \%$ respectivamente).

El $4 \%$ eran analfabetos, $14 \%$ sin estudios, $44,2 \%$ estudios de primer grado, 25,6\% estudios de segundo grado y $11,6 \%$ estudios de tercer grado. En cuanto al estado civil: $60,5 \%$ casados, $18,6 \%$ solteros, $18,6 \%$ viudos y $2,3 \%$ separado/divorciado. La mayoría de los pacientes convivía con su familia (79\%), un $14 \%$ vivía solo y un $7 \%$ en una institución sociosanitaria. Respecto al nivel de ingresos mensuales, un $16,3 \%$ recibía menos de $500 €, 51,2 \%$ entre 500 y $1000 €$, $11,6 \%$ entre 1000 y $1500 €, 16,3 \%$ entre 1500 $2000 €$ y $4,7 \%$ más de $2000 €$. 
En los gráficos 1 a 3 se muestran la descripción del estado de salud para la totalidad de la muestra según las 5 dimensiones del EQ-5D. La puntuación media de la EVA fue de 56,84 \pm 20 puntos (rango 12-96). En el gráfico 4 se muestra la percepción del estado de salud con respecto a los últimos 12 meses para la totalidad de la muestra.

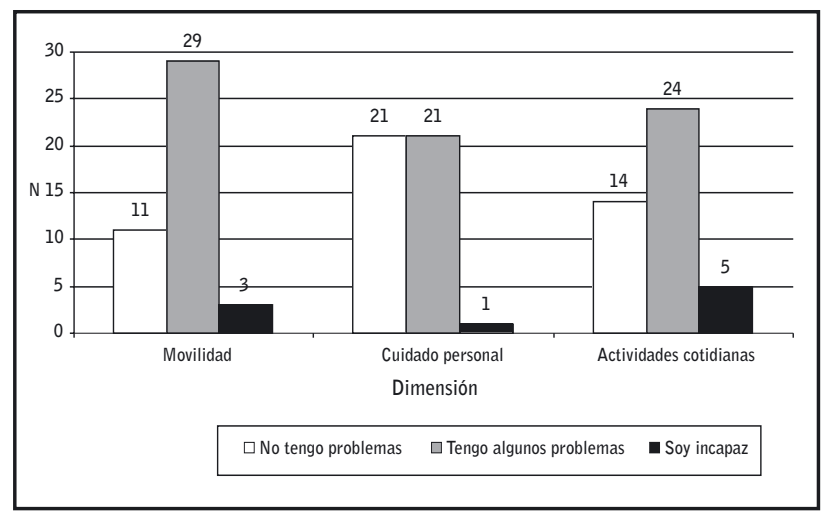

Gráfico 1: Evaluación de las dimensiones movilidad, cuidado personal y actividades cotidianas del EQ5D para la totalidad de la muestra.

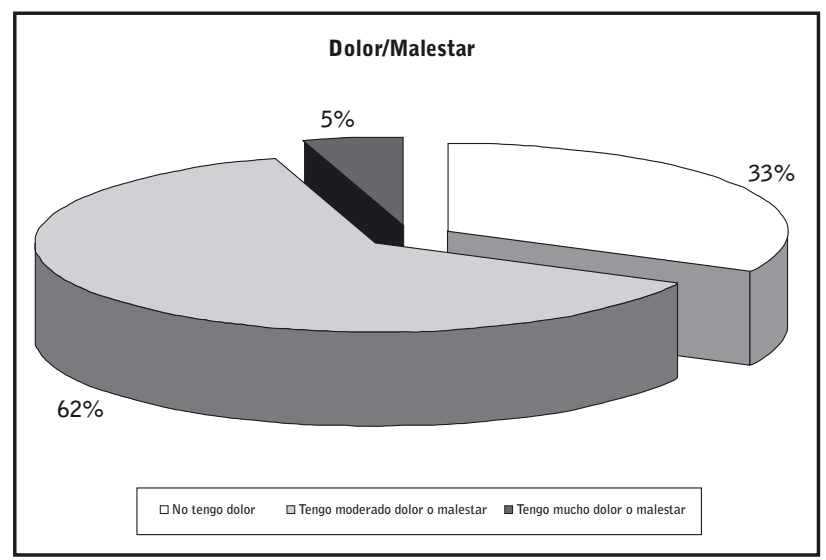

Gráfico 2: Evaluación respecto a la dimensión dolor/ malestar del EQ-5D para la totalidad de la muestra.

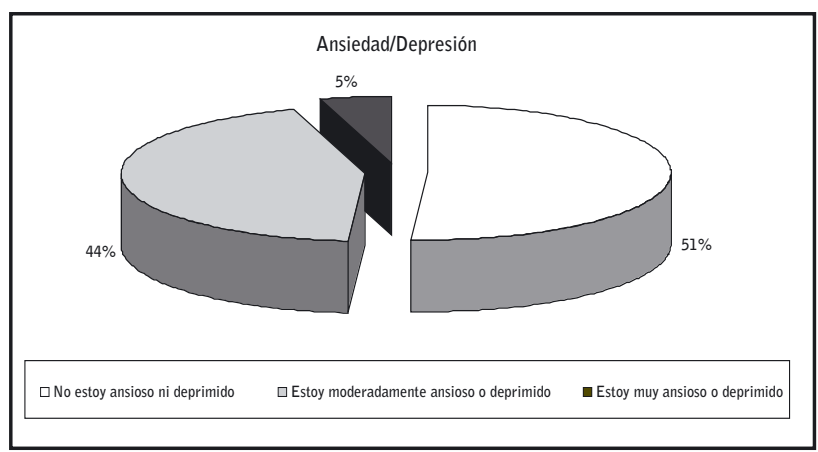

Gráfico 3: Evaluación respecto a la dimensión ansiedad/ depresión del EQ-5D para la totalidad de la muestra.

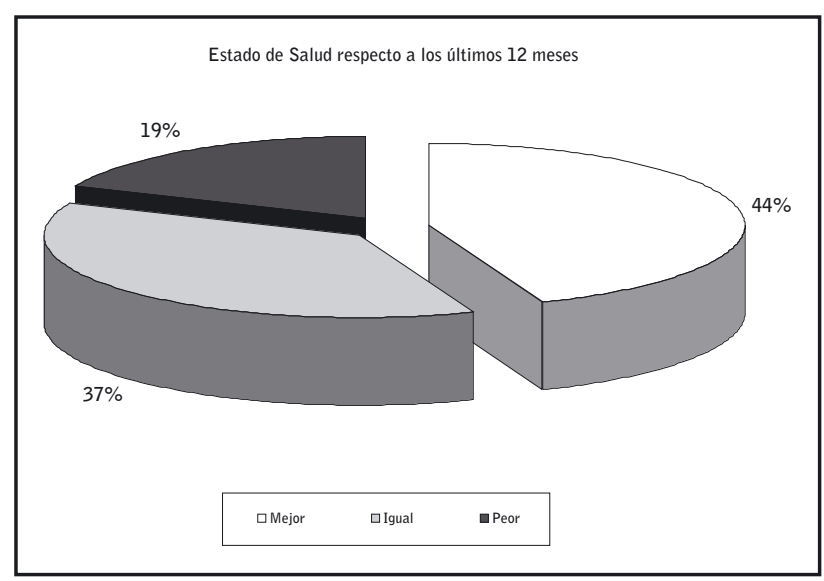

Gráfico 4: Percepción del estado de salud comparado con los últimos 12 meses para la totalidad de la muestra.

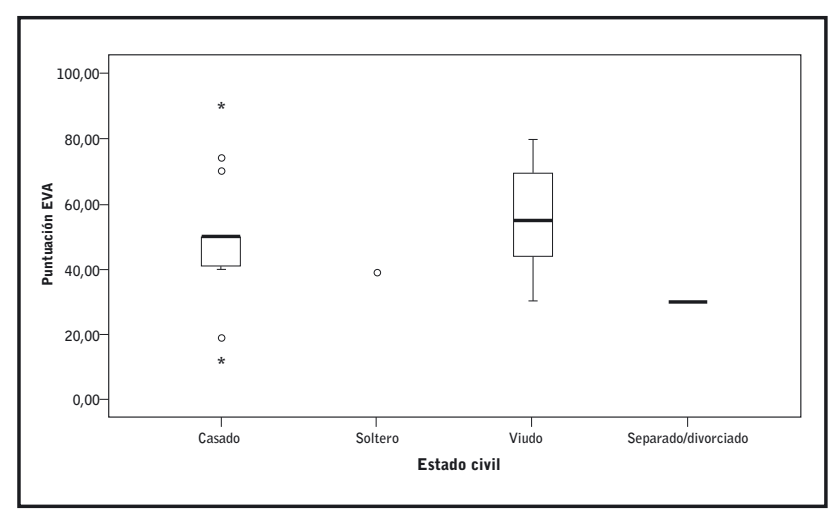

Gráfico 5: Puntuación de la escala visual analógica (EVA) del EQ-5D en función del estado civil del paciente

El nivel de estudios no se correlacionó de forma estadística con ninguna de las dimensiones del EQ-5D ni con la puntuación de la EVA.

El estado civil se correlacionó con la puntuación de la EVA $(p=0,012)$, puntuando mejor los solteros $(33,94$ puntos) frente a los casados (19,17 puntos), los viudos (21,56 puntos) y los separados (3,5 puntos) (gráfico5). También se correlacionó con un mayor grado de ansie$\mathrm{dad} /$ depresión $(\mathrm{p}=0,002)$, presentando mayores niveles de ansiedad/depresión los casados (moderadamente ansioso/deprimido 42,3\%, muy ansioso o deprimido $3,8 \%$ ) frente a los solteros y viudos (moderadamente ansioso/deprimido $50 \%$ respectivamente) y los separados/divorciados ( $100 \%$ muy ansiosos/deprimidos). En cuanto a la percepción de su estado de salud respecto 
a los últimos 12 meses, el estado civil también tuvo su influencia $(p=0,016)$, teniendo una mejor percepción los solteros (mejor $87,5 \%$, peor $12,5 \%$ ) que los casados (mejor 38,5\%, igual 30,8\%, peor 30,8 \%), que los viudos (mejor $25 \%$, peor $75 \%$ ) y que los separados/ divorciados ( $100 \%$ peor).

El tipo de convivencia tuvo relación, aunque no significativa $(p=0,062)$, con la puntuación de la EVA, considerando mejor su estado de salud aquellos que vivían solos $(31,5$ puntos) frente a los que vivían con su familia (21,19 puntos) y en una institución sociosanitaria (12,17 puntos) (gráfico 6). Aquellos que vivían solos presentaban menores problemas con las actividades cotidianas (no tengo problemas $83,3 \%$, algunos problemas $16,7 \%$ ), que los que vivían en familia (no tengo problemas $26,5 \%$, algunos problemas $58,8 \%$, soy incapaz $14,7 \%$ ) y los que vivían en una institución sociosanitaria (algunos problemas $100 \%)(p=0,037)$. Los que vivían en familia presentaban mayor dolor/malestar (moderado 70,6\%, mucho 5,9\%) que los que vivían en una institución sociosanitaria (moderado 66,7\%, mucho $0 \%$ ) y solos (moderado 16,7\%, mucho 0\%), pero sin llegar a ser estadísticamente significativo $(p=0,075)$.

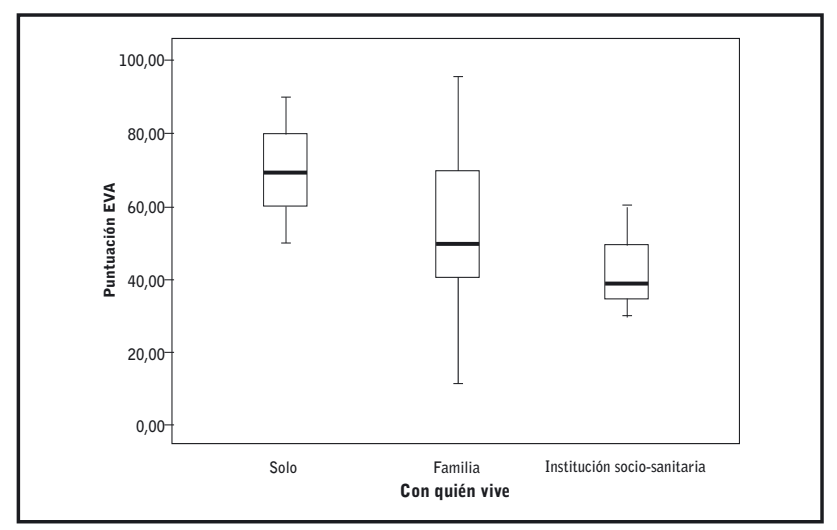

Gráfico 6: Puntuación de la escala visual analógica EVA del EQ-5D en función del tipo de convivencia del paciente

El nivel de ingresos mensuales se correlacionó de forma inversa con problemas en el cuidado personal $(p=0,016)$, el $100 \%$ de los que ingresaban menos de $500 €$ tenía algunos problemas; entre 500 y 1000 $€$ el $45 \%$ tenía algunos problemas; entre 1000 y 1500 €en $40 \%$ tenía algunos problemas y el $20 \%$ era incapaz; entre $1500-2000 €$ el $14,3 \%$ tenía algunos problemas. La edad se correlacionó con una peor percepción del estado de salud respecto a los últimos 12 meses $(p=0,006)$, siendo los mayores de 65 años los que peor percepción tenían (60\% peor, $20 \%$ igual, $20 \%$ mejor) frente a los menores de 65 años ( $17,4 \%$ peor, $17,4 \%$ mejor, $65,2 \%$ mejor) (gráfico 12). El sexo no se correlacionó de forma estadísticamente significativa con ninguno de los parámetros del EQ-5D.

\section{Discusión}

Las puntuaciones generales en todas las dimensiones del EQ-5D de nuestro estudio son mucho mejores que las presentadas por Raimundo y cols $^{7}$, donde el $80 \%$ presentaban muchos problemas relacionados con el autocuidado y actividades cotidianas, un $57 \%$ en la movilidad y un $47 \%$ referían su estado de salud como peor comparado con el último año. Nuestras puntuaciones se asemejan más a los resultados del grupo de Wit ${ }^{10}$, que encontraron una asociación negativa entre la edad y el dominio físico del cuestionario SF36 y con la puntuación de la EVA, como ocurre en nuestro estudio. La edad ha sido uno de los parámetros más estudiados en cuanto a la CVRS y los pacientes en HD. Baltar y cols ${ }^{11}$ observaron que los pacientes mayores de 65 años obtuvieron puntuaciones significativamente mayores (peor CVRS) que los menores de esa edad, en las dimensiones del área física del Perfil de Consecuencias de la Enfermedad (PCE): funcionamiento físico, rol físico, dolor y salud general. Las variables que demostraron influir de forma independiente sobre la puntuación del PCE fueron: la mayor edad (aunque hay que recordar que las puntuaciones del PCE no están estandarizadas); el sexo femenino; la puntuación en la Escala de Karnofsky menor o igual a 70; el mayor índice de comorbilidad; y el nivel de educación bajo.

En nuestro caso ni el sexo ni el nivel educativo se correlacionaron con una peor percepción del estado de salud.

Rebollo y cols ${ }^{12}$ en un estudio sobre 170 pacientes en $H D$, vieron que las puntuaciones del cuestionario SF-36 eran mayores (mejor CVRS) para los pacientes jóvenes en cinco dimensiones: funcionamiento físico, dolor, vitalidad, funcionamiento social y salud mental, siendo esta diferencia estadísticamente significativa en la dimensión funcionamiento físico. En nuestro caso los mayores de 65 años presentaban peor percepción 
de su estado de salud comparado con los últimos 12 meses. Brown y cols ${ }^{13}$ inciden en su revisión acerca de la necesidad de cuidados paliativos en los pacientes en HD mayores de 65 años, ya que los estudios revisados demuestran que son los que peor CV tienen, y que los cuidados paliativos mejoran la percepción de su estado de salud.

Parece que lo que más influía en la CVRS de nuestros pacientes es con quien conviven, asociándose con peores puntuaciones de la EVA, peor percepción del estado de salud en los últimos 12 meses y más problemas de depresión, de aquellos que viven en una institución sociosanitaria y en familia, que los que viven solos. Esto se puede deber a que los que viven solos son menos dependientes en cuanto a las actividades de la vida diaria, lo que hace lógicamente tengan una mejor CVRS. El estado civil también se asoció con peor CVRS, sobre todo en cuanto a la depresión/ansiedad. Los casados refieren peores puntuaciones en la EVA y peor estado de salud percibido en el último año, probablemente debido a que por su enfermedad se hacen más dependientes de sus esposa-os. Kao y cols ${ }^{14}$ estudiaron 861 pacientes en HD observaron que unos ingresos mensuales más altos y el aumento de las actividades sociales están asociados con la mejora de la CVRS, mientras que una mayor preocupación y puntuaciones más altas del Beck Depresion Inventory se asocian con peor CVRS de los pacientes en HD. En nuestro caso, el nivel de ingresos mensuales se correlacionó de forma inversa con problemas en el cuidado personal. Para el grupo de Muñoz ${ }^{15}$, la CVRS al año de comenzar en HD viene determinada de forma significativa por el estado de depresión, la edad y el grado de comorbilidad, siendo las responsables del $67 \%$ de la variabilidad del dominio función física del SF36. Los pacientes de Barrena y cols también presentaban peor CVRS a mayor edad, hasta los 80 años, que tendían a mejorar de nuevo ${ }^{16}$.

Una limitación de nuestro estudio ha sido no tener en cuenta el grado de comorbilidad de los pacientes estudiados, que aunque no era objeto de nuestro estudio, influye de manera directa sobre la CVRS.

\section{Conclusiones}

Los mayores problemas relacionados con la CVRS de los pacientes en HD se refieren a la movilidad y actividades cotidianas. Casi el $70 \%$ de los pacientes presenta dolor/malestar y casi la mitad se encuentran ansiosos o deprimidos. Las variables sociológicas que más influyen en la CVRS de los pacientes en HD son la edad, el estado civil, la convivencia y el nivel de ingresos. Variables modificables como el nivel de ingresos puede hacer que disminuyan estas desigualdades en salud entre pacientes con una misma patología, dotando de más recursos a los pacientes en HD. Los casados presentan peor CVRS probablemente relacionado con que la enfermedad les hace ser más dependientes de sus parejas.

Recibido: Febrero 2011

Revisado: Febrero 2011

Modificado: Marzo 2011

Aceptado: Abril 2011

\section{Bibliografía}

1. Casas J, Repullo JR, Pereira J. Medidas de calidad de vida relacionada con la salud. Conceptos básicos, construcción y adaptación cultural. Med Clin (Barc) 2001; 116: 789-796.

2. WHOQOL Group. Study protocol for the World Health Organization Project to develop a quality of life assessment instrument. Qual Life Res. 1993; 2(2):153-9.

3. Tobita I, Hyde C. Quality of life research: a valuable tool for nephrology nurses. J Ren Care 2007; 33(1):25-9.

4. Ruiz de Alegría-Fernández de Retana $B$, BasabeBarañano N, Fernández-Prado E, Baños-Baños C, Nogales-Rodríguez MA, Echavarri-Escribano M y cols. Calidad de vida y afrontamiento: diferencias entre los pacientes de diálisis peritoneal continua ambulatoria y hemodiálisis hospitalaria. Enferm Clin 2009; 19 (2):61-8.

5. Cukor D, Cohen SD, Peterson RA, Kimmel PL. Psychosocial aspects of chronic disease: ESRD as a paradigmatic illness. J Am Soc Nephrol. 2007; 18(12):3042-55. 
6. Badia X, Schiaffino A, Alonso J, Herdman M. Using the EuroQoI 5-D in the Catalan general population: feasibility and construct validity. Qual Life Res. 1998; 7(4): 311- 22.

7. Raimundo P, Ravasco P, Proença V, Camilo M. Does nutrition play a role in the quality of life of patients under chronic haemodialysis? Nutr Hosp. 2006; 21(2):139-44.

8. Hessel FP, Mitzner SR, Rief J, Gress S, Guellstorff $B$, Wasem J. Economic evaluation of MARS--preliminary results on survival and quality of life. Liver. 2002; 22 (Suppl 2):26-9.

9. Tyrrell J, Paturel L, Cadec B, Capezzali E, Poussin $G$. Older patients undergoing dialysis treatment: cognitive functioning, depressive mood and healthrelated quality of life. Aging Ment Health. 2005; 9(4):374-9.

10. De Wit GA, Merkus MP, Krediet RT, de Charro FT. Health profiles and health preferences of dialysis patients. Nephrol Dial Transplant. 2002; 17(1):8692.

11. Baltar J, Ortega F, Rebollo P, Alvarez-ude F, Caramelo C, Valdés F, Álvarez Grande J, Badía X. Salud percibida en pacientes mayores de 65 años en trata- miento sustitutivo renal. Nefrología 1999; 19(Supl 1):73-83.

12. Rebollo P, González MP, Bobes J, Saiz P, Ortega F. Interpretación de los resultados de la calidad de vida relacionada con la salud de pacientes en terapia sustitutiva de la insuficiencia renal terminal. Nefrologia 2000; 20(5):431-9.

13. Brown EA. Epidemiology of renal palliative care. J Palliat Med 2007; 10(6):1248-1252.

14. Kao TW, Lai MS, Tsai TJ, Jan CF, Chie WC, Chen WY. Economic, social, and psychological factors associated with health-related quality of life of chronic hemodialysis patients in northern Taiwan: a multicenter study. Artif Organs. 2009; 33(1):61-8.

15. Muñoz Sancho R, Oto Royo A, Barrio Alonso R, Fernández M. Evolución de la calidad de vida en pacientes en hemodiálisis: Estudio prospectivo a un año. Rev Soc Esp Enferm Nefrol 2006; 9 (1):55-58.

16. Barrena Solaguren $E$, Goiricelaya Indurain $A$, Menica Jáuregui I, Hernández López J, Mayor Iturburaga JM. Percepción de la calidad de vida en el paciente de hemodiálisis. Libro de Comunicaciones Presentadas al XXXIII Congreso Nacional SEDEN. Madrid: Hospal; 2008. 